\title{
Additive manufacturing in maxillofacial reconstruction
}

\author{
Luciana Laura Dincă ${ }^{1, *}$, Alexandra Banu $^{1}$ and Aurelian Vișan ${ }^{1}$ \\ ${ }^{1}$ Politehnica University of Bucharest, Department of Manufacturing Technology, Splaiul \\ Independenţei no. 313, sector 6, Bucharest, RO-060042, Romania
}

\begin{abstract}
In this paper the benefits of using additive manufacturing technologies in maxillofacial reconstruction are highlighted. Based on a real clinical case, the paper describes the manufacture of an implant prototype replacing the right zygomatic bone and a part of maxilla using additive manufacturing technologies. The face is the most expressive part of the human body that makes us unique. It was shown that the maxillofacial prostheses help to improve the psychological state of patients affected by, because low self esteem feeling appears commonly to this patients with the facial defects. The aim of this paper is to show how using additive manufacturing technologies methods within this research, the producing a surgical model will help surgeon to improve the pre-operative planning. For this we used additive manufacturing technologies such as Stereolitography to achieve the biomodel and FDM-fused deposition modelling to obtain a prototype model because these technologies make it possible to obtain prosthesis according to the physical and mechanical requirements of the region of implantation.
\end{abstract}

\section{Introduction}

Facial defects create psychological effects resulting in a low self-confidence due to their appearance. Because of many factors such as: complexity of the face's bones, the vital adjacent structures, the possibility of infection and because of the uniqueness of the each patient, the manufacturing of maxillofacial implant is a challenge for engineers to design a accurate implant but also is a challenge for doctors to help the patients to look like before [1].

In medicine additive manufacturing technologies are used in different way: for the production of end-use implants, models and moulds usually are used SLS and FDM technologies, for the production of models, moulds and surgical guides are used SLA technology, and SLM and EBM for the production of end-use implants [3].

A method of producing a precise model using 3D printing which can facilitate preoperative planning is shown in a D. J. Thomas et al. study [4], in which they developed a method of obtaining 3D printed model of mandible highlighting that the surgical procedures reduce risk and also the patient and hospital expenses.

\footnotetext{
*Corresponding author: lucianalaura_shamieh@yahoo.com
} 
Another study which highlights the benefits of producing the models is that developed by Anni Suomalainen et al. [5] in which had been shown that models facilitate and improve the treatment planning.

The use of CAD / CAM systems have made it possible to design and manufacture customized implants. CAS-computer assisted surgery technique, brings together the use of several technologies such as: Conversion of scans from Computed Tomography, CT; Nuclear Magnetic Resonance, MRI; Rapid Prototyping, RP; 3D CAD design; Finite Element Analysis, FEA; Rapid Manufacturing, RM; Reverse Engineering; Robotics. The most important advantage in CAS techniques is the precision of the patterns resulting from the use of this technique. Specialized imaging techniques from CT, MRI in 3D models can be easily used by doctors to see the defect from any point, so CAS techniques help the doctor to better understand the anatomy involved by viewing and simulating [6].

In maxillofacial reconstruction, these technologies have gained a special place due to the possibility to obtain a perfect implant from patient data.

The main objective of our paper is to highlight the benefits of using additive manufacturing in maxillofacial reconstruction. This study started from a tomographic images of the patient 46 years old male affected by maxillofacial cancer, after which zygomatic bone and a part of maxilla were removed. In the implants manufacturing area, additive manufacturing technologies opens a lot of possibility. The paper is focusing on the steps of obtaining biomodel and the implant prototype using these technologies.

\section{Experimental details}

\subsection{Materials}

To fabricate the biomodel, patient data was required. The curable material used to obtain the biomodel in the SLA- stereolithography method was an acrylate.

To obtain the implant prototype we used FDM-fused deposition modeling method and material was an plastic ABS -filament.

\subsection{Method}

The biomodel and the prototype were made after CT images obtained by Toshiba MRI equipment under the following conditions: 6,47mA, 920 slices and $0.2 \mathrm{~mm}$ layer thickness. We used SLA -stereolithography method involving the use of an uv beam to solidify a liquid photopolymer resin in the printer construction drum. The stereolithography equipment which we used to obtain the biomodel was from SLA-250 series. This equipment allows the construction of complicated conceptual models. Due to the high precision and speed of high quality workpiece formation, the SLA-250 machines are successfully used in the medical field.

Manufacturing of implant prototype was performed with the FDM-fused deposition modeling method. We used Zmorph equipment to obtain the implant prototype.

\section{Results and discussions}

\subsection{Design prototype- general principes}

To elaborate this paper Mimics software was used, which is a program that helps to convert DICOM images in 3D model and also to design the implant. MIMICS Materialise is a 
useful viewing and segmenting tool for scanned images from CT or MRI and 3D rendering of images, and more Mimics can be used to diagnose and plan the surgery [7].

The development and design of customized implants is important in order to minimize the possibility of achieving an implant that does not fit the implantation region [8]. There are different methods for designing the custom implant, but the design technique should be taken into account to provide more accurate results and fitting the implants to the place of implantation as much as possible.

As a result of the Moiduddin K, et.al. (2016) study [9], the "mirror" technique offers more precision in the case of medium (20 to $40 \mathrm{~mm}$ ) and large (41 to $53 \mathrm{~mm}$ ) sized tumors compared to the "anatomical reconstruction" technique. For small sized tumors $(<20 \mathrm{~mm})$ for the reconstruction of maxillofacial implants it is recommended to use the anatomical reconstruction technique. Having the virtual 3D model resulted from conversion of DICOM images into 3D model help us to design implant prototype using the mirror imaging technique, after chose correctly sagittal plane, because of nonsimetry of the patient head. Because additive process offers no limits to design issues or complexities, using additive manufacturing technologies such as SLA - stereolithography and FDM - fused deposition modeling we have obtained the biomodel and the implant prototype.

\subsection{The steps in obtaining the implant prototype}

At the beginning, the patient went for CT scan to obtain the latest facial morphology. The CT scan shows that in the right side the zygomatic bone and a part of maxilla area are missing (figure 1).

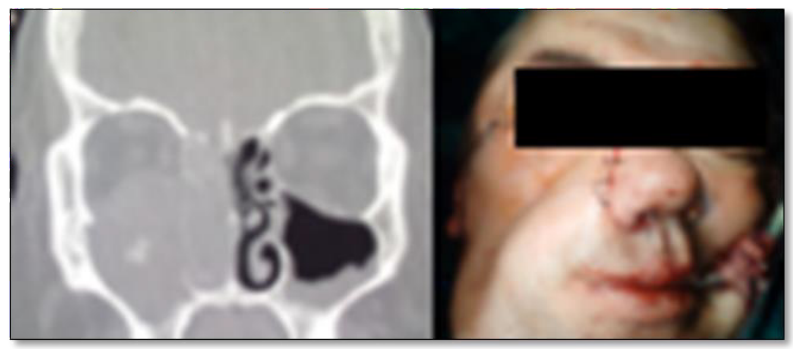

Fig. 1. CT scan confirming the defect and clinical appearance of the patient.

The CT data DICOM was imported into medical imaging software MIMICS, for 3D reconstruction (figure 2).

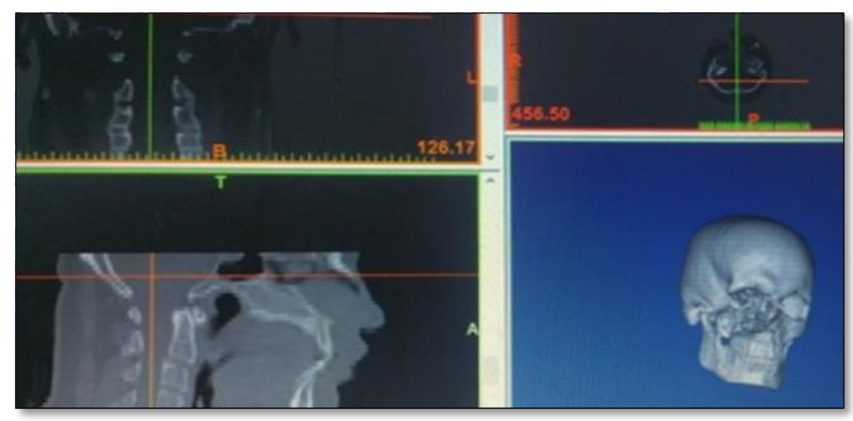

Fig. 2. The reconstruction model-missing part. 


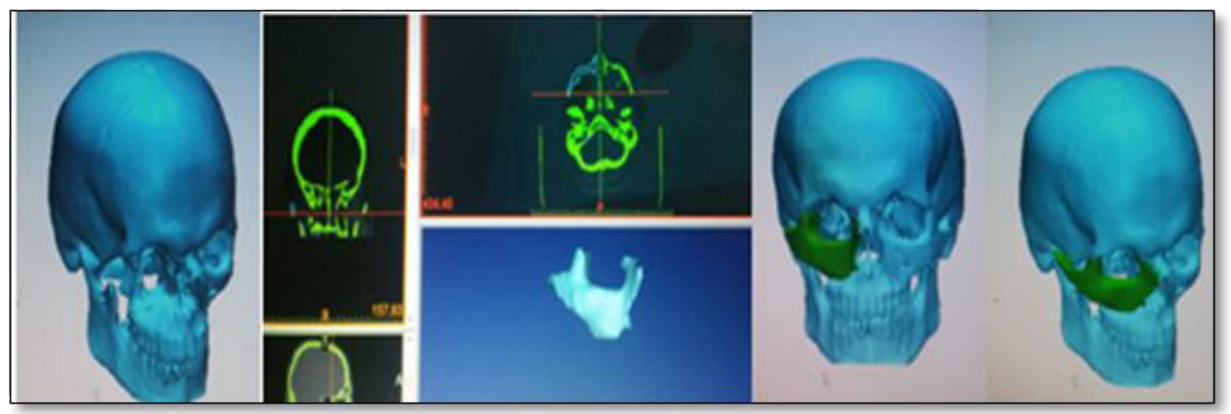

Fig. 3. The implant prototype design steps-the virtual implant.

After this, the next step was to save into stl format and fabricate them, using additive manufacturing technologies. To create the biomodel we used stereolithography, which is a forming additive manufacturing which product part layer by layer using photopolymerization. It is well known that one of the advantages of this technologies is its speed. For biomodel printing time was 16 hours, because of the complexity and because additional support structures were necessary to be built in the beginning of the process. The difficulty we have met when we had to remove the additional structures after process was finised (figure 4).

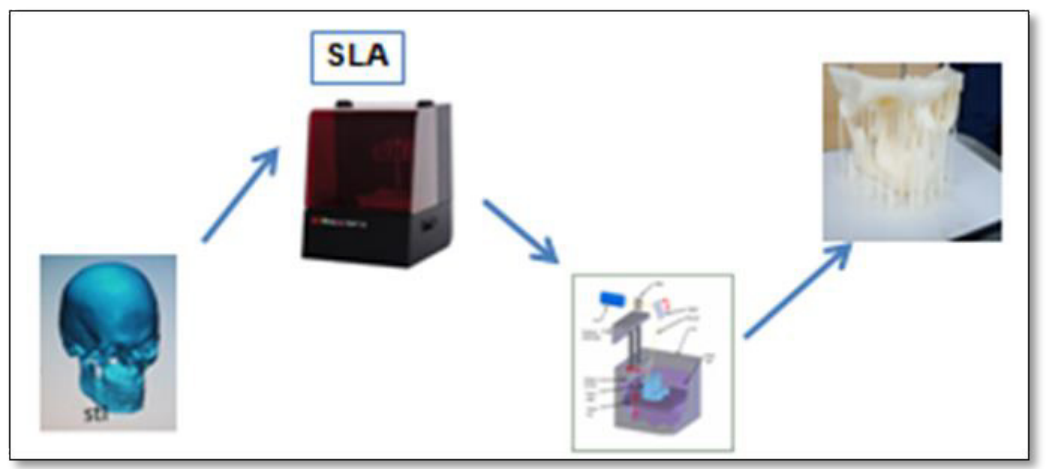

Fig. 4. The SLA manufacturing process of biomodel.

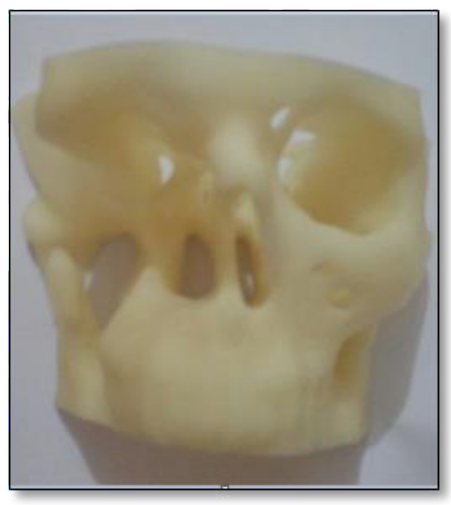

Fig. 5. The patient biomodel manufactured by SLA.

To design the implant we used mirror technique. On the sagittal plane, half of the face, covering the defect area was removed and mirrored the left side.

In the FDM-fused deposition modeling method case from stl format the plastic melt layer after layer, in the implant designed stl shape (figure 6). The thickness layer was 
$0.2 \mathrm{~mm}$. For our implant, were necessary additional vertical support structures, because of the irregular shape.

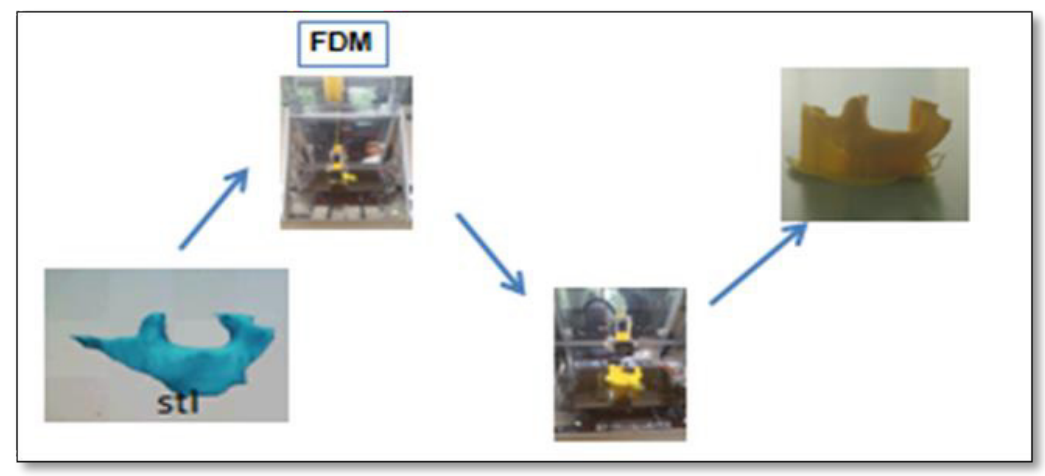

Fig. 6. The FDM manufacturing process of implant prototype.

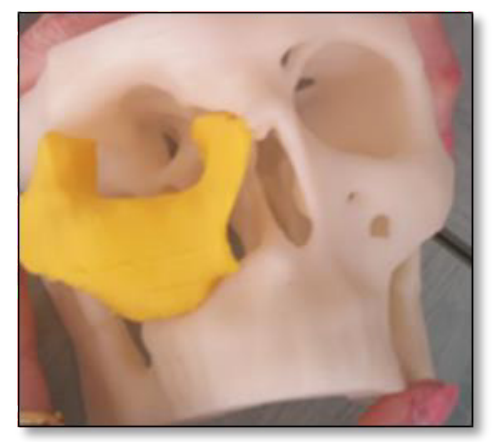

Fig. 7. The implant prototype and biomodel manufactured by additive manufacturing technologies.

Accurate life-sized 3D implant prototype was successfully created for our patient, using additive manufacturing technologies. Fabricating the implant prototype helps us to understand how will be fixed. So the implant model can facilitate the pre-operative planning and make it posible the selection of the most appropriate implant. The next implant will be made of titanium, using also the additive manufacturing technologies, that is SLM- selective laser melting. The titanium implant that we aim to achieve will have a role in restoring the aesthetic appearance, has a support function for attaching the muscle, a protective role for environmental factors, and will also have a support function for a silicone prosthesis, giving the patient a natural look.

\section{Conclusions}

Additive manufacturing technologies provided the benefits in obtaining the accurate implants. Using these technologies helps us to improve our 3D understanding of anatomy, and how the implant will be fixed. The opportunity to see the model from different angles in a natural way is a new way in medical diagnosis and treatment. This medical model will help surgeon to improve their pre-operative planning of the patient. 


\section{References}

1. J. Parthasarathy, Annals of Maxillofacial Surgery 4 (1), 9 (2014)

2. K. Kitsakis, Z. Moza, V. Iakovakis, N. Mastorakis, J. Kechagias, An Investigation of Dimensional Accuracy of Multi-Jet Modeling Parts MMMAS 2015 International Conference on Mathematical Models and Methods in Applied Sciences (151-157). Agios Nikolaos, Crete, Greece (2015)

3. A. Thompson, D. McNally, I. Maskery and R. K. Leach, Int. J. Metrol. Qual. Eng. 8, 17 (2017)

4. D. J. Thomas et al. Int J Adv Manuf Technol 71, 1643 (2014)

5. A. Suomalainen et al., J Clin Exp Dent. 7 (5), e605 (2015)

6. H. Kawachi, et al., Bull Tokyo Dent Coll. 51 (1), 35 (2010)

7. C. Sadhasivam, S. K. Gunda., International Journal of Science and Research 4 (7), (2015)

8. K. Moiduddin, et al., Technol Health Care 24 (3), 377 (2016)

9. K. Moiduddin, et al., International Journal of Advanced Biotechnology and Research 7 (1), 323- (2016) 